\title{
Epidemiology, Serogroups and Resistance of Salmonella During a I5-Year Period (2006-2020) in Kuwait
}

\author{
Wafaa Jamal' \\ Fatema Bibi Khodakhast' \\ Manuel John Albert' \\ Vincent Rotimi ${ }^{2}$ \\ 'Department of Microbiology, Faculty of \\ Medicine, Kuwait University, Jabriya, \\ Kuwait; ${ }^{2}$ Department of Medical \\ Microbiology and Parasitology, College of \\ Medicine, Lagos State University, Ikeja, \\ Nigeria
}

Purpose: The aim of the study was to investigate the changing pattern in serogroup distribution and antimicrobial resistance of all Salmonella spp. isolated from patients attending the Mubarak Al Kabeer Hospital (MAK), Kuwait from 2006 to 2020.

Patients and Methods: A retrospective study of all enrolled patients attending the MAK with culture-positive Salmonella spp. was undertaken. Data on age, gender, culture sample and serogroup were obtained from the laboratory information system. A prospective antimicrobial susceptibility of all stock isolates was carried out using E test. The trend rates of Salmonella serogroups and antimicrobial resistance were compared among 5 periods: 20062008, 2009-2011, 2012-2014, 2015-2017, and 2018-2020.

Results: A total of 700 isolates were identified. The majority of the isolates were from the stool (77.6\%), followed by the blood (16.4\%). The most common serogroups were serogroup D (37.6\%) and B (23.4\%). There was a significant rise in ciprofloxacin resistance from $32.2 \%$ during $2006-2008$ to $54.3 \%$ during $2018-2020$ and from $32.5 \%$ during $2009-2011$ to $54.3 \%$ during $2018-2020$ ( $P=0.0001$, respectively). The resistance trend to cefotaxime was at relatively low levels ranging from $0 \%$ to $3.4 \%$ through $2006-2008$ to $2018-2020$. There was a significant drop of the resistance to ampicillin from $23.6 \%$ in $2015-2017$ to $12.3 \%$ in 2006-2008 to 2018-2020 ( $P=0.03)$. Trimethoprim/sulfamethoxazole resistance dropped significantly from 14.5 to $3.6 \%(P=0.002)$ during $2006-2008$ to $2018-2020$ and then from 13.5 to $3.6 \%(P=0.02)$ during $2015-2017$ to $2018-2020$. One hundred and seventeen $(16.7 \%)$ isolates were multidrug-resistant.

Conclusion: Continuous surveillance of Salmonella and its antimicrobial resistance is important for antibiotic policy formulation for invasive Salmonella infections.

Keywords: salmonella, susceptibility, serogroups, resistance, state of Kuwait
Correspondence: Wafaa Jamal Department of Microbiology, Faculty of Medicine, Kuwait University, PO

Box 24923, Safat, Jabriya, 13110, Kuwait

Tel +96524633509

Fax +96525332719

Email wafaa.jamal@ku.edu.kw

\section{Introduction}

Salmonellae are motile, Gram-negative bacilli, belonging to the family Enterobacterale. The genus Salmonella consists of 3 species: Salmonella bongori, Salmonella enterica and S. subterranean. ${ }^{1}$ S. bongori (subspecies V) causes diseases in reptiles and rarely causes disease in humans, while $S$. enterica consists of over 2600 serotypes or serovars that have been identified up to date and causes disease in humans. ${ }^{1} S$. enterica itself is composed of six subspecies: entericae (subspecies I), salamae (subspecies II), arizonae (subspecies IIIa), diarizonae (subspecies IIIb), houtanae (subspecies IV), indica (subspecies VI). ${ }^{1}$ S. enterica serovar is divided into typhoidal and nontyphoidal strains. Almost all serotypes can cause disease in 
humans. A few are host-specific and can be found in few animal species, eg $S$. enterica serotype Dublin in cattle and S. enterica serotype Choleraesuis in pigs. ${ }^{2}$

Salmonella infection is an important cause of foodborne-disease and gastroenteritis worldwide. According to the World Health Organization (WHO), it is regarded as 1 of the 4 key global causes of diarrheal diseases. ${ }^{2}$ Most cases are mild but some can be life threatening according to the host factors and serotype of salmonella. It causes a considerable morbidity and mortality, especially in developing countries. ${ }^{3}$ WHO has estimated that every year, almost 1 out of 10 people become sick and 33 million of healthy life years are lost. The Centers for Disease Control and Prevention (CDC), USA estimates that approximately 1.35 million illnesses, 26,500 hospitalizations and 420 deaths occur due to non-typhoidal Salmonella each year in the US, resulting in an estimated $\$ 400$ million in direct medical costs. ${ }^{4}$

There is no vaccine available for controlling invasive non-typhoidal Salmonella infection. Although antimicrobial agents are not recommended in the treatment of salmonella gastroenteritis, it is, however, recommended for extra-intestinal invasive infections, especially in the extreme of age, immunosuppressed patients and those with underlying diseases, eg, meningitis, septicemia, septic arthritis, and osteomyelitis. ${ }^{5,6}$ The management of these invasive infections entails using antimicrobial agents. However, antimicrobial resistance in Salmonella spp. is a serious issue all over the world especially against the first-line drugs, eg, ampicillin, chloramphenicol, and cotrimoxazole. Third-generation cephalosporins and fluoroquinolones have become the standard first-line empirical therapy. A CDC report stated that antibiotic-resistant nontyphoidal Salmonella infections are on the rise approaching an estimated $10 \%$ for ciprofloxacin, $3 \%$ for ceftriaxone and $1 \%$ for azithromycin. ${ }^{4}$ Prolonged hospitalization and increased risk of bloodstream infections, treatment failure and excess mortality have been associated with antimicrobial drug resistant non-typhoidal Salmonella infections. ${ }^{6-9}$ Previous reports from Kuwait have shown that resistance to Salmonella spp. in Kuwait is high. ${ }^{10,11}$ According to a previous report from Kuwait, the commonest serogroups in both adults and children were serogroup B followed by serogroup $\mathrm{C}$ and D. ${ }^{9}$ Thus, it is important to do a continuous surveillance of salmonella antimicrobial resistance and serogroups for patient management in order to reduce the occurrence of complications and mortality.
The aim of this study was to investigate the changing pattern in serogroup distribution and antibiotic resistance among Salmonella isolates from patients attending the Mubarak Al Kabeer Teaching Hospital, Kuwait, during a period of 15 years from 2006 to 2020 .

\section{Materials and Methods}

\section{Bacterial Isolates}

All Salmonella species isolated from inpatients and outpatients attending the Mubarak Al Kabeer Hospital from 2006 to 2020 were sent to Anaerobe/Hospital Infection Laboratory, Department of Microbiology, Faculty of Medicine, Kuwait University, where they were stored at $-80^{\circ} \mathrm{C}$ freezer in CryoBank beads (Mast Group Limited, Merseyside, UK). The specimens were obtained from the following sites as requested by the clinical diagnosis: stool, blood, extraintestinal samples, eg, urine, CSF, pus, tissue, pleural fluid, bed sore, synovial fluid, bile, eye swab, ascetic fluid, wound, and fine needle aspirates. Only one isolate per patient per site was collected. The isolates were stored at $-80^{\circ} \mathrm{C}$ freezer as above until used for study. Data on patient's age, gender, culture site, date of collection and serogroups were obtained from the laboratory information system (LIS).

\section{Culture Method}

Stool specimens were cultured on the following media: MacConkey agar (Oxoid, Basingstoke, UK), SalmonellaShigella (SS) agar (ThermoFisher Scientific, Waltham, MA, USA), Campylobacter agar (ThermoFisher Scientific) and Selenite F broth (Becton Dickinson, Franklin Lakes, NJ, USA) which was subcultured on SS gar after 18 $\mathrm{h}$ incubation. If the stool was watery, it was also cultured on Aeromonas selective agar (Sigma-Aldrich, St. Louis, MO, USA) and Sorbitol-MacConkey agar (Oxoid). ${ }^{12}$

The following automated blood culture systems were used: BACTEC 9240 (Becton Dickinson) from 2006 to 2014, and BD BACTEC FX (Becton Dickinson) and BACT/ALERT VIRTUO (bioMérieux, Marcy-l'Etoile, France) from 2015.

\section{Identification and Serogrouping}

All Salmonella isolates were re-identified and serogrouped by standard VITEK II system (bioMerieux) and slide agglutination test during 2021. Isolates with low scores on VITEK II were subjected to further identification on Matrix-Assisted Laser Desorption/Ionization-Time of Flight (MALDI-TOF) (bioMerieux). For serogrouping, the isolates were inoculated into triple sugar iron (TSI) agar (Hardy Diagnostics, Santa 
Maria, CA, USA) and slide agglutination test was carried out using Salmonella polyvalent and group-specific antisera (Denka Seiken, Tokyo, Japan; SSI Diagnostics, Hillerod, Denmark; and Remel, Thermo Fisher Scientific) according to manufacturer instructions.

\section{Antimicrobial Susceptibility Testing}

Susceptibility testing of all isolates was carried out during 2021, in the Anaerobe/Hospital Infection Laboratory by determining the minimum inhibitory concentrations (MIC). The antibiotics tested and their breakpoints were the following: ampicillin $(8 \mu \mathrm{g} / \mathrm{mL})$, cefotaxime $(1 \mu \mathrm{g} / \mathrm{mL})$, chloramphenicol $(8 \mu \mathrm{g} / \mathrm{mL})$, ciprofloxacin $(0.06 \mu \mathrm{g} / \mathrm{mL})$, ertapenem $(0.5 \mu \mathrm{g} /$ $\mathrm{mL})$, gentamicin $(4 \mu \mathrm{g} / \mathrm{mL})$, meropenem $(1 \mu \mathrm{g} / \mathrm{mL})$, tigecycline $(0.5 \mu \mathrm{g} / \mathrm{mL})$ and trimethoprim/sulfamethoxazole $(2 / 38$ $\mu \mathrm{g} / \mathrm{mL}$ ) using E-test method (bioMerieux). The breakpoints were used according to the interpretive criteria recommended by the Clinical and Laboratory Standards Institute (CLSI), except for tigecycline, which was done according to The European Committee on Antimicrobial Susceptibility Testing (EUCAST) ${ }^{13,14}$ Escherichia coli ATCC 25922 strain was used as a quality control strain in each batch of test.

To look for changes in resistance over time, the duration of study was broken into five periods: A (2006 to 2008), B (2009 to 2011), C (2012 to 2014), D (2015 to 2017), and E (2018 to 2020).

\section{Statistical Analysis}

The difference between proportions was compared by a two-tailed Chi square test. A $P$ value of $\leq 0.05$ was considered as significant.

\section{Ethical Approval}

Institutional ethical approval was obtained from the Health Sciences Centre Ethical Committee, Health Sciences Centre, Kuwait University (permit number VDR/EC/3779). Collection of the specimens was conducted according to the Declaration of Helsinki and with particular institutional ethical and professional standards. No additional specimens were collected from the patients for this study and the patient identities were kept anonymous.

\section{Results}

\section{Demographics and Epidemiology}

As shown in Table 1, a total of 700 isolates were collected of which 543 (77.6\%) were from stool, 115 (16.4\%) from blood, 18 (2.6\%) from urine, $1(0.1 \%)$ from cerebrospinal fluid (CSF), and 21 (3\%) from other extra-intestinal sites (7 isolates from pus, 4 tissue, 2 pleural fluids, 2 ascetic fluids, 1 bedsore, 1 synovial fluid, 1 bile, 1 eye swab, 1 wound and 1 fine needle aspirate). The most common serogroup was group D (263; $37.6 \%)$, followed by group B $(164 ; 23.4 \%)$, group C $(154$; $22 \%)$, Salmonella Typhi (46; 6.5\%), group E (30; 4.3\%), miscellaneous groups $(17 ; 2.4 \%)$, group $\mathrm{G}(14 ; 2 \%)$, and Salmonella Paratyphi A, B, and C (12; 1.7\%). The miscellaneous group include the following: group $\mathrm{F}(6 ; 0.85 \%)$, group A $(3 ; 0.4 \%)$, group I $(2 ; 0.28 \%)$, group O $(2 ; 0.28 \%)$, group J (2; $0.28 \%)$, group H $(1 ; 0.14 \%)$, and Salmonella enterica subsp. diarizonae $(1 ; 0.14 \%)$. Among the bloodstream infections, group D $(45 ; 39.1 \%)$ was the commonest followed by $S$. Typhi $(40 ; 34.8 \%)$, group B $(11 ; 9.6 \%)$ and $S$. Paratyphi A, $\mathrm{B}$ and $\mathrm{C}(6 ; 5.2 \%)$ and group C $(6 ; 5.2 \%)$. Only one case of meningitis due to Salmonella serogroup D was reported during

Table I Clinical Samples from Which Salmonella Was Isolated

\begin{tabular}{|c|c|c|c|c|c|c|c|}
\hline \multirow[t]{2}{*}{ Salmonella Groups/Species } & \multicolumn{5}{|c|}{ No. of Isolates in } & \multirow[b]{2}{*}{ Unknown Samples } & \multirow[t]{2}{*}{ Total No. of Isolates } \\
\hline & Stool & Blood & Urine & CSF & Other Samples ${ }^{b}$ & & \\
\hline Salmonella grp. B & 140 & 11 & 3 & 0 & 9 & I & 164 \\
\hline Salmonella grp. C & 140 & 6 & 1 & 0 & 6 & 1 & 154 \\
\hline Salmonella grp. D & 203 & 45 & 10 & I & 4 & 0 & 263 \\
\hline Salmonella grp. E & 27 & 1 & 2 & 0 & 0 & 0 & 30 \\
\hline Salmonella grp. G & 11 & 3 & 0 & 0 & 0 & 0 & 14 \\
\hline Salmonella Typhi & 4 & 40 & 2 & 0 & 0 & 0 & 46 \\
\hline Salmonella Paratyphi (A, B, C) & 6 & 6 & 0 & 0 & 0 & 0 & 12 \\
\hline Miscellaneous ${ }^{\mathbf{a}}$ & 12 & 3 & 0 & 0 & 2 & 0 & 17 \\
\hline Total Salmonella spp. & 543 & 115 & 18 & 1 & 21 & 2 & 700 \\
\hline
\end{tabular}

Notes: a Miscellaneous: Salmonella group F (6); Salmonella group A (3); Salmonella group I (2); Salmonella group O (2); Salmonella group J (2); Salmonella group H (I); and

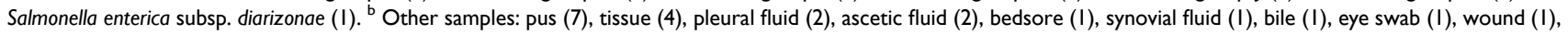
and fine needle aspirate (I).

Abbreviation: CSF, cerebrospinal fluid. 
Table 2 Age and Gender Distribution of Patients from Whom Salmonella Was Isolated

\begin{tabular}{|c|c|c|c|c|c|}
\hline \multirow[t]{2}{*}{ Salmonella Group/Species } & \multirow[t]{2}{*}{ Total No. of Isolates } & \multicolumn{2}{|c|}{ Gender } & \multicolumn{2}{|c|}{ Age $^{b}$} \\
\hline & & Male & Female & Mean (Years) & Range \\
\hline Total Salmonella spp. ${ }^{\mathrm{a}}$ & 700 & 393 & 306 & 28.3 & 23 Days- $90 Y$ \\
\hline Salmonella grp. $\mathrm{D}^{\mathrm{a}}$ & 263 & $15 \mid$ & 111 & 28.6 & 23 Days - 90Y \\
\hline Salmonella grp. B & 164 & 91 & 73 & 30.2 & 3 months- $86 Y$ \\
\hline Salmonella grp. C & 154 & 86 & 68 & 30.3 & I month - $78 \mathrm{Y}$ \\
\hline Salmonella Typhi & 46 & 23 & 23 & 18.6 & $2 Y-75 Y$ \\
\hline Salmonella grp. E & 30 & 14 & 16 & 29.6 & 6 months- $46 Y$ \\
\hline Miscellaneous & 17 & 9 & 8 & 23 & I Y $-80 Y$ \\
\hline Salmonella grp. G & 14 & II & 3 & 16 & $\mid Y-5 I Y$ \\
\hline Salmonella Paratyphi & 12 & 7 & 5 & 19.7 & II months $-44 \mathrm{Y}$ \\
\hline
\end{tabular}

Notes: ${ }^{a}$ Gender was unknown for one sample. ${ }^{b}$ Ages were not available for: Total Salmonella spp. (232), S. group B (70); S. group C (55); S. group D (49); S. Typhi (23); S. group E (19); miscellaneous group (5); S. group G (9); S. Paratyphi (2).

Abbreviations: $M$, month; $Y$, years.

the study period. As shown in Table 2, more than half of the isolates were obtained from male patients $(393 ; 56.2 \%)$ compared to $(306 ; 43.9 \%)$ from female patients. The age ranged between 23 days to 90 years. The age stratification according to the groups is shown in Table 2.

\section{Changing Trends in Resistance Rates Among Different Years}

As shown in Figure 1, there was a significant drop in the isolation rates of resistant isolates against ampicillin from $23.6 \%$ to $12.3 \%(P=0.03)$ between periods 2015-2017 and 2018-2020, respectively. While trimethoprim/sulfamethoxazole resistance dropped significantly from 14.5 to $3.6 \% \quad(P=0.002)$ for periods between 2006-2008 and 2018-2020 and from 13.5 to $3.6 \%(P=0.02)$ for periods $2015-2017$ and $2018-2020$. There was a significant rise in resistance to gentamicin from 3.5\% during 2006-2008 to $10.1 \%$ during $2015-$ $2017(P=0.05)$. There was a significant rise in ciprofloxacin resistance from $32.2 \%$ during $2006-2008$ to $54.3 \%$ in $2018-2020$, and again from $32.5 \%$ during 2009-2011 to $54.3 \%$ in $2018-2020 \quad(P=0.0001$ for both comparisons). Tigecycline resistance dropped significantly from $60.5 \%$ during $2006-2008$ to $31.9 \%$ during 2018-2020, from 52.6\% during 2009-2011 to $31.9 \%$ during $2018-2020$, and from $59.6 \%$ during $2015-2017$ to $31.9 \%$ in $2018-2020 \quad(P=0.0001$ for all three comparisons) and from $59.6 \%$ during period 2012-2014 to $31.9 \%$ during $2018-2020 \quad(P=0.0008)$. Third-generation cephalosporins, cefotaxime, had an excellent activity against all isolates with resistance rates ranging from $0 \%$ to $3.4 \%$ during 2015-2017.
Meropenem and ertapenem demonstrated excellent activities against all isolates with $0 \%$ resistance in the 15 -year study period. Resistance to chloramphenicol was relatively high at $10.5 \%$ during the initial 6 years, then dropped by nearly one-third $(3.4 \%$ to $3.9 \%$ ) and finally spiked to $5.1 \%$ during the last 3 years of the study.

\section{Resistance Rates for Different Years for Different Salmonella Groups Against}

\section{Ampicillin}

As shown in Figure 2, there was a significant drop in ampicillin resistance in Salmonella group B from 27.5\% to $10.6 \%$ in $2006-2008$ to $2009-2011 \quad(P=0.03)$. But there was a significant rise in resistance for group D from $11.1 \%$ during 2009-2011 to $28.6 \%$ during 2015-2017 $(P=0.02)$ followed by a significant drop from $28.6 \%$ for the period 2015-2017 to $11.4 \%$ in $2018-2020(P=0.02)$.

\section{Resistance Rates of Different Salmonella Groups Against Ciprofloxacin in Different Years}

As shown in Figure 3, isolates belonging to Salmonella Group D demonstrated a significant rise of ciprofloxacin resistance from $27.6 \%$ during $2006-2008$ to $67.1 \%$ during 2018-2020 ( $P=0.0004)$, from $17.5 \% \quad(2009-2011)$ to $40.7 \%$ (2012-2014) $(P=0.03)$, from $17.5 \%$ in $2009-2011$ to $67.1 \%$ in $2018-2020(P=0.01)$, and from $39.3 \%$ (2015$2017)$ to $67.1 \%(2018-2020)(P=0.002)$. There were no significant changes in resistance pattern for other groups. 


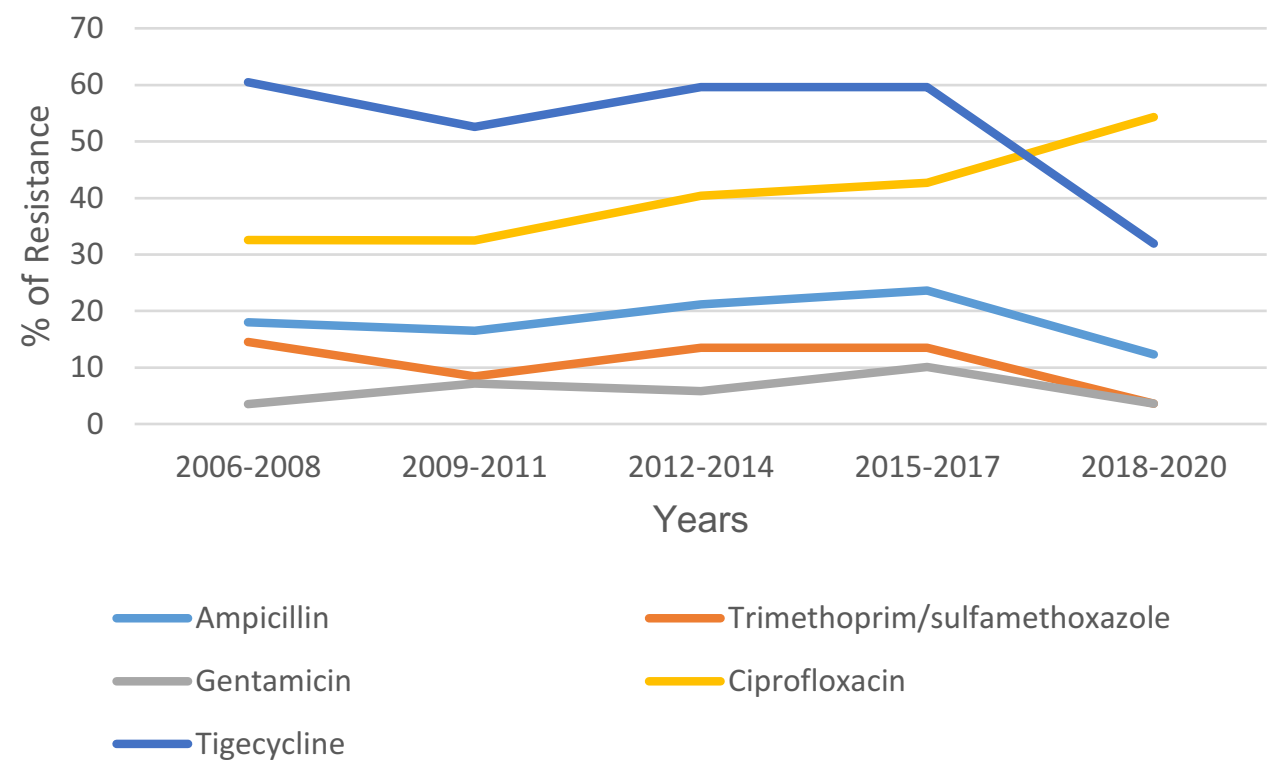

Figure I Resistance of all Salmonella groups during different years. Significant difference between periods for prevalence of resistance for antibiotics is as follows: 2015-2017 vs 2018-2020 for ampicillin ( $P=0.03)$; 2006-2008 vs $2018-2020(P=0.002)$ and 2015-2017 vs 2018-2020 ( $P=0.02)$ for trimethoprim; $2006-2008$ vs $2015-2018$ ( $P=0.05)$ for gentamicin; $2006-2008$ vs $2018-2020(P=0.000 I)$ and $2009-201$ I vs $2018-2020$ ( $P=0.0001)$ for ciprofloxacin; 2006-2008 vs $2018-2020$ ( $P=0.000$ I), 2009-20II vs $2018-$ $2020(P=0.0001), 2015-2017$ vs $2018-2020(P=0.0001)$, and $2012-2014$ vs $2018-2020(P=0.0008)$ for tigecycline.

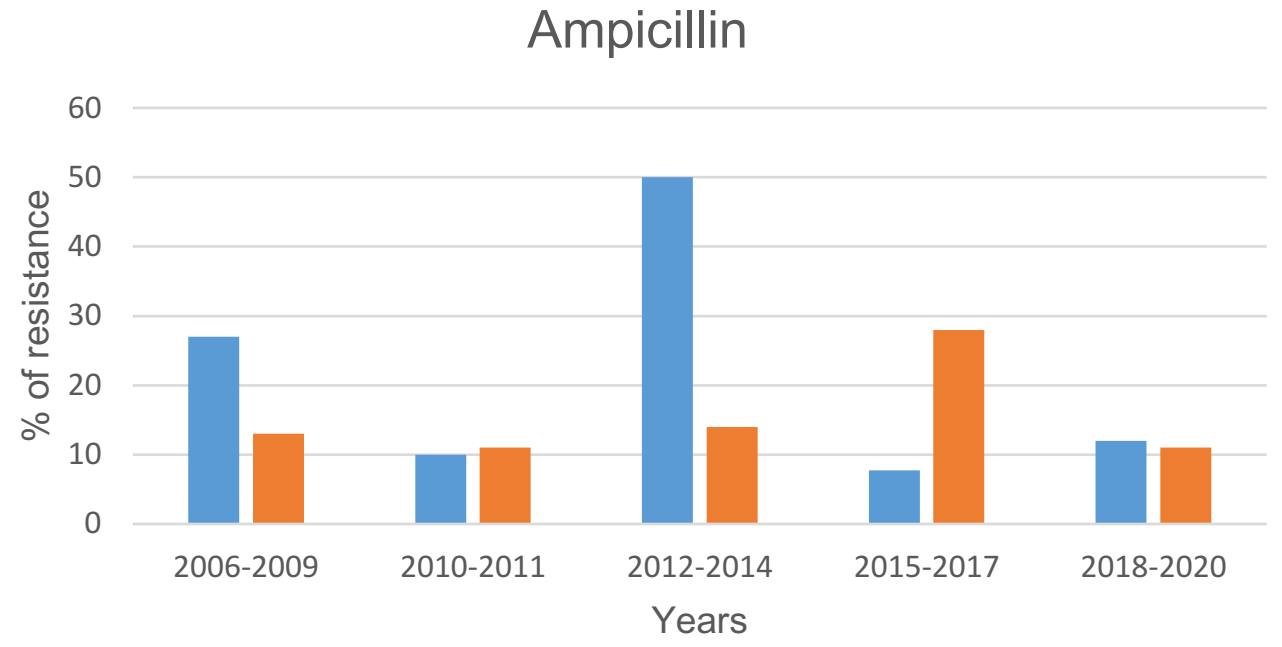

Group B (no. 164) Group D (no. 263)

Figure 2 Resistance of Salmonella groups B and D for the five periods against ampicillin. Significant difference between periods for prevalence of resistance for ampicillin is as follows: $2006-2008$ vs $2009-201$ I $(P=0.03)$ in Salmonella group B; and 2009-20II vs 2015-2017 $(P=0.02)$ and 2015-2017 vs $2018-2020$ ( $P=0.02)$ in Salmonella group D.

\section{Resistance Rates for Different Years for Different Salmonella Groups Against}

\section{Tigecycline}

As shown in Figure 4, there was a significant drop of resistance to tigecycline in Salmonella group B from $76.5 \%(2006-2008)$ to $56.1(2009-2011)(P=0.031)$ and from $76.5 \% \quad(2006-2008)$ to $41.7 \% \quad(2018-2020)$
$(P=0.005)$. Among Salmonella group $\mathrm{C}$, the resistance rate to tigecycline dropped significantly from $77.5 \%$ (2006-2008) to $65.3 \%(2009-2011)(P=0.002)$ and from $65.3 \%$ (2009-2011) to $33.3 \%$ (2018-2020) $(P=0.01)$. Among Salmonella group D, the resistance level increased significantly from $44.8 \%$ (2006-2008) to $47.6 \%$ (20092011) $(P=0.04)$. However, the resistance level dropped 


\section{(Salmonella Group D )}

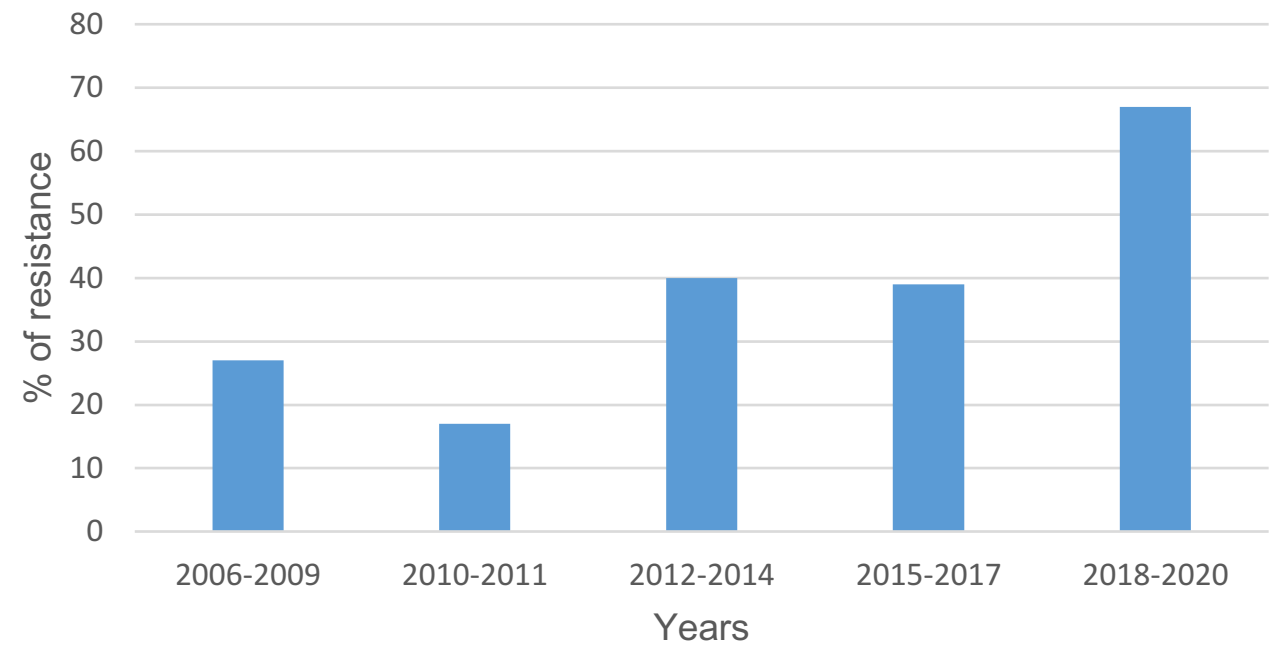

Figure 3 Resistance of Salmonella group D for the five periods against ciprofloxacin. Significant difference between periods for prevalence of resistance for ciprofloxacin is as

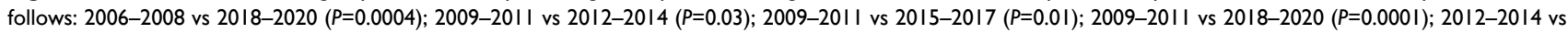
20I5-2017 $(P=0.02)$; and 2015-2017 vs 2018-2020 $(P=0.0002)$.

\section{Tigecycline}

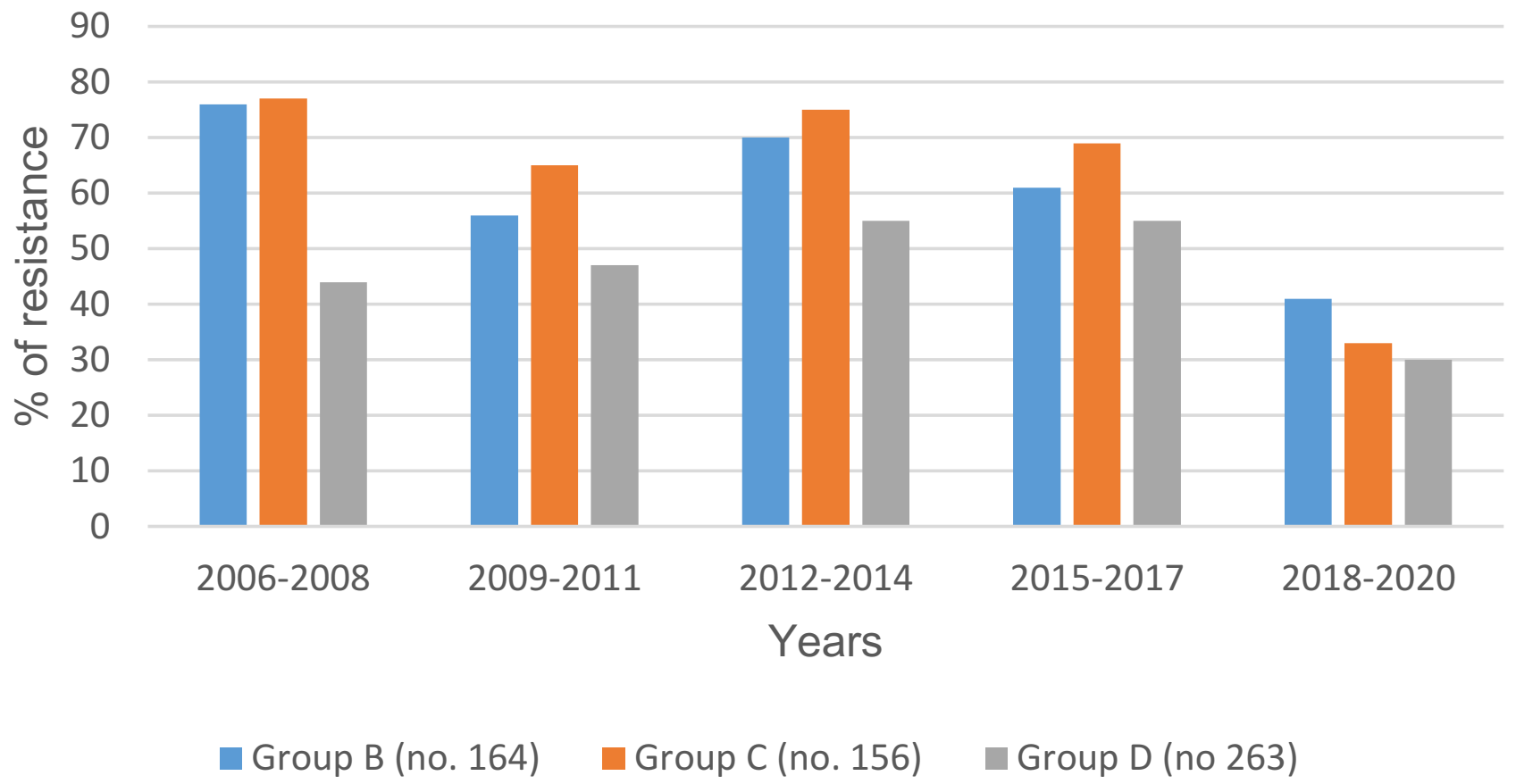

Figure 4 Resistance of Salmonella groups $B, C$ and $D$ for the five periods against tigecycline. Significant difference between periods for prevalence of resistance for tigecycline is as follows: $2006-2008$ vs $2009-201$ I $(P=0.03)$ and $2006-2008$ vs $2018-2020(P=0.0005)$ in Salmonella group B; 2006-2009 vs 20I8-2020 ( $P=0.002)$ and 2009$201 \mathrm{I}$ vs 20I8-2020 ( $P=0.01)$ in Salmonella group $C$; and 2006-2008 vs $2018-2020(P=0.04), 2012-2014$ and $2018-2020(P=0.02)$ and $2015-2017$ vs $2018-2020(P=0.005)$ in Salmonella group D. 
significantly from $55.6 \%$ (2012-2015) to $30.7 \%$ (20182020) $(P=0.02)$ and from $55.4 \%(2015-2017)$ to $30.7 \%$ (2018-2020) $(P=0.0005)$.

\section{Resistance Rates for Different Years for Different Salmonella Groups Against Chloramphenicol, Trimethoprim/ Sulfamethoxazole, Gentamicin and Cefotaxime}

There were no significant differences in the resistance rates for chloramphenicol, trimethoprim/sulfamethoxazole, gentamicin and cefotaxime in different years for the different salmonella groups (data not shown).

\section{Multidrug Resistance Phenotypes}

The resistance phenotypes of all 700 isolates are shown in Table 3. A total of 492 isolates $(70.28 \%)$ were resistant to one or more antibiotics. One hundred and seventeen isolates (16.7\%) were multidrug-resistant isolates (ie, resistant to 3 or more different classes of antibiotics). Fifty-one (7.2\%), 48 $(6.85 \%), 14(2 \%)$, and $4(0.57 \%)$ isolates were resistant to 3 , 4, 5 and 6 antimicrobial agents, respectively. Insight into the origin of multi-resistant strains is given in Table S1. There were 27 patterns of multi-resistance. The most prevalent patterns were: ampicillin, ciprofloxacin, and tigecycline (16 isolates) $>$ ampicillin, gentamicin, ciprofloxacin, and tigecycline ( 15 isolates) $>$ chloramphenicol, ampicillin, and ciprofloxacin (11 isolates) > chloramphenicol, ampicillin, trimethoprim-sulfamethoxazole, and ciprofloxacin (10 isolates). The involvement of antibiotics in multi-resistant was tigecycline (22 isolates) $>$ ampicillin and ciprofloxacin (20 isolates) $>$ trimethoprim-sulfamethoxazole (15 isolates) $>$ chloramphenicol (14 isolates) $>$ gentamicin (13 isolates). Many multi-resistant isolates were spread across Salmonella groups B, C and D. Multi-resistance strains were present across all five periods.

\section{Discussion}

Salmonella infections are an important public health issue all over the world. There is a geographic difference in distribution of Salmonella serogroups in children and adults in different countries including Kuwait. In a previous study from Kuwait, it was reported that serogroup B was more prevalent than serogroups C. ${ }^{10}$ However, in this study, serogroup D was the most prevalent followed by serogroup B, a finding similar to previous reports from Saudi Arabia, Turkey, Taiwan, and Malawi. ${ }^{15-19}$
Table 3 Resistance Phenotypes of All 700 Salmonella Isolates

\begin{tabular}{|c|c|}
\hline Resistance Phenotypes & No. of Isolates \\
\hline TGC & 173 \\
\hline Cip & 82 \\
\hline Cip, TGC & 79 \\
\hline Amp, Cip, TGC & 16 \\
\hline Amp, Gn, Cip, TGC & 15 \\
\hline Chl, Amp, Cip & 11 \\
\hline Chl, Amp, TS, Cip & 10 \\
\hline Amp, TGC & 9 \\
\hline TS, TGC & 8 \\
\hline TS, Cip, TGC & 8 \\
\hline Amp & 7 \\
\hline Chl, Cip, TGC & 6 \\
\hline Amp, TS, Cip, TGC & 6 \\
\hline Amp, Cip & 5 \\
\hline Amp, TS, TGC & 5 \\
\hline Chl, Amp, TS, TGC & 5 \\
\hline TS, Cip & 4 \\
\hline Chl, Amp, Cip, TGC & 4 \\
\hline Amp, TS, Gn, Cip, TGC & 4 \\
\hline Amp, TS & 3 \\
\hline Chl, Amp, TS, Gn, TGC & 3 \\
\hline Chl, TS, Gn, Cip, TGC & 3 \\
\hline Chl, Amp, TS, Gn, Cip, TGC & 3 \\
\hline Chl, TS, TGC & 2 \\
\hline Amp, Gn, Cip & 2 \\
\hline Gn, Cip, TGC & 2 \\
\hline Chl, Amp, CT, Cip & 2 \\
\hline Amp, TS, Gn, TGC & 2 \\
\hline Amp, CT, Cip, TGC & 2 \\
\hline Chl, Amp, Gn, Cip, TGC & 2 \\
\hline $\mathrm{Chl}$ & 1 \\
\hline Chl, TGC & I \\
\hline Amp, CTX & 1 \\
\hline Amp, Gn, TGC & I \\
\hline TS, Gn, Cip, TGC & I \\
\hline Chl, TS, Gn, Cip & I \\
\hline Chl, Amp, TS, Cip, TGC & I \\
\hline Amp, Gn, CT, Cip, TGC & I \\
\hline Chl, Amp, TS, Gn, CT, TGC & I \\
\hline
\end{tabular}

Abbreviations: Chl, chloramphenicol; Amp, ampicillin; Cip, ciprofloxacin; TGC, tigecycline; TS, trimethoprim/sulfamethoxazole; CTX, cefotaxime; Gn, gentamicin.

Our Salmonella isolates demonstrated a relative drop in the resistance to the first-line drugs during the 15-year period. For instance, resistance to chloramphenicol dropped from $10.5 \%$ to $5 \%$, likewise resistance to ampicillin (18\% to $12.3 \%)$ and trimethoprim/sulfamethoxazole (14.5\% to $3.6 \%)$. This observation may be explained, in part, by the fact that there has been steady replacement of the conventional drugs by the quinolones and thirdgeneration cephalosporins over time in our hospitals and 
community. Our observation is concordant with a similar report from Europe where there was a decline in the occurrence of resistance to chloramphenicol from $14 \%$ to $8 \%{ }^{20}$ However, unlike our finding of decline in resistance to the first-line drugs, reports from Saudi Arabia and Taiwan have actually demonstrated an increase in resistance to the first-line drugs. ${ }^{15,18}$

Resistance to the third-generation cephalosporin such as cefotaxime and ceftriaxone had been reported in Salmonella spp. since $1991 .^{21}$ This resistance appeared to be due to plasmid mediated AmpC or ESBL genes. In a study reported from Kuwait, CTX-M-15 with insertion sequence ISEcpl gene was identified in several Salmonella serotypes belonging to serogroups B and C. ${ }^{22}$ In the current study, although cefotaxime resistance was detected in $0.4 \%, 3.6 \%$ and $3.4 \%$ during 2009-2011, 2015-2017, and 2018-2020, respectively, there were no significant differences in the resistance during these 3 periods. In the current study, resistance to cefotaxime was lower than previously reported in Kuwait, Saudi Arabia and Europe. ${ }^{15,20,22}$ However, this is unlike a previous study from Taiwan, where the resistance to cefotaxime increased from $<5 \%$ to $>10 \%$ in serogroup B and S. Choleraesuis from 1999 to $2010 .^{23}$

In our study, there was a gradual increase in ciprofloxacin resistance from $32.2 \%$ to $54.3 \%$ during the study period and there was a significant rise in resistance between periods of 2006-2008 vs 2018-2020, and 2009-2011 vs 2018-2020. This rise in the ciprofloxacin resistance is of concern as it is the drug of choice in Kuwait to treat invasive salmonella infections. Ciprofloxacin resistance is mediated by mutations in $g y r A$ and $g y r B$ genes that lead to mutations in the quinolone resistance determining region. ${ }^{24}$ This probably explains our observation as there has been a persistent increase in the use and misuse of this drug in the healthcare centers. It is even mandatory to give people on pilgrimage to holy sites in Saudi Arabia a capsule of the antibiotic as a prophylaxis against communicable diseases. These measures exert undue pressure on the bacteria to develop resistance.

Carbapenem is the drug of choice for treating patients with MDR-salmonella infections when the thirdgeneration cephalosporin and quinolones are ineffective. Although none of our isolate, in this study, was resistant to carbapenems, resistance to this group of antibiotics has been reported previously. ${ }^{25}$ Should resistance to this class of drugs develop in the future, it would further complicate the treatment of invasive salmonella infections.
It was noted that tigecycline resistance dropped during the 15 -year period from $60.5 \%$ to $31.9 \%$ with statistically significant differences among several periods. This is unlike the situation in Taiwan in 2008, where $1.6 \%$ of Salmonella serotype Typhimurium isolates, and $1.6 \%$ of Salmonella serotype Choleraesuis isolates were not susceptible to tigecycline using the EUCAST breakpoints. ${ }^{26}$ The reason for this high resistance level among our isolates is probably due to the overuse of tigecycline at the onset of its introduction to the country, but whose use gradually tapered off with time and development of resistance among other species of the family Enterobacterales. ${ }^{27}$

Since the 1990s, the prevalence of multidrug-resistant Salmonella spp. has been on the increase worldwide, including Kuwait, the UK, and USA. ${ }^{11,28,29}$ Our previous report in 2008 showed that 28 out of 287 isolates were MDR representing $9.8 \%$ of the total isolates. In this report, the MDR rate was $16.7 \%$, nearly doubling over the previous rate. ${ }^{11}$ This observation is discordant with the report from Turkey, Taiwan and Europe where the rate of MDR dropped over time. ${ }^{16,18,20}$ Multi-resistant strains were present in several groups and in many periods. This is not surprising since the antibiotics tested were in use for treatment before we started the study.

Although the susceptibility testing of the isolates was prospective, the patient data collection was retrospective with its own limitations. Other limitations include: involvement of a single center, lack of access to clinical data including mortality and morbidity rates, and absence of investigation of molecular mechanisms of resistance in the resistant isolates.

\section{Conclusion}

There was a significant rise in ciprofloxacin resistance and a significant drop in ampicillin and trimethoprimsulfamethoxazole resistances in Salmonella during the 15year study period. Resistance to cefotaxime was low and steady, and multi-resistance was below $20 \%$. Continuous surveillance of Salmonella and its antimicrobial resistance provided information on these changing trends. This type of study is required for formulation of antibiotic policy to treat serious invasive infections.

\section{Abbreviations}

MAK, Mubarak Al Kabeer Hospital; WHO, World Health Organization; CDC, Center for Disease Control and Prevention; MALDI-TOF, matrix-assisted laser desorption/ ionization-time of flight; TSI, triple sugar iron; MIC, 
minimum inhibitory concentration; EUCAST, European Committee on Antimicrobial Susceptibility Testing.

\section{Disclosure}

All authors declare no competing interests in this work.

\section{References}

1. Su LH, Chin C-H. Salmonella: clinical importance and evolution of nomenclature. Chang Gung Med J. 2007;30(3):210-219.

2. WHO. Salmonella (non-typhoidal); 2021. Available from: https:// www.who.int/news-room/fact-sheets/detail/salmonella-(nontyphoidal). Accessed November 18, 2021.

3. Majowicz SE, Musto J, Scallan E, et al. The global burden of nontyphoidal Salmonella gastroenteritis. Clin Infect Dis. 2010;50 (6):882-889. doi:10.1086/650733

4. CDC. Antibiotic Resistance Threats in the United States, 2019. Atlanta, GA: U.S. Department of Health and Human Services, CDC; 2019.

5. Gordon MA. Invasive non-typhoidal Salmonella disease- epidemiology, pathogenesis and diagnosis. Curr Opin Infect Dis. 2011;24 (5):484-489. doi:10.1097/QCO.0b013e32834a9980

6. Gilchrist JJ, Maclennan CA. Invasive nontyphoidal Salmonella disease in Africa. EcolSalPlus. 2019;8(2):254.

7. Verma JK, Molbak K, Barrett TJ, et al. Antimicrobial-resistant nontyphoidal Salmonella is associated with excess bloodstream infections and hospitalizations. J Infect Dis. 2005;191(4):554-561. doi:10.1086/427263

8. Helms M, Vastrup P, Gerner-Smidt P, Molbak K. Excess mortality associated with antimicrobial drug-resistant Salmonella typhimurium. Emerg Infect Dis. 2002;8(5):490-495. doi:10.3201/eid0805.010267

9. Molbak K, Baggesen DL, Aarestrup FM, et al. An Outbreak of Multidrug-Resistant, Quinolone-Resistant Salmonella enterica Serotype Typhimurium DT104. $N$ Engl J Med. 1999;341 (19):1420-1425. doi:10.1056/NEJM199911043411902

10. Jamal WY, Pal T, Rotimi VO, Chugh TD. Serogroups and antimicrobial susceptibility of clinical isolates of Salmonella species from a teaching hospital in Kuwait. J Diarrhoeal Dis Res. 1998;16 (3):180-186.

11. Rotimi VO, Jamal W, Pal T, Sonnevend A, Dimitrov TS, John Albert M. Emergence of multidrug-resistant Salmonella species and isolates with reduced susceptibility to ciprofloxacin in Kuwait and the United Arab Emirates. Diag Microbiol Infect Dis. 2008;60(1):71-77. doi:10.1016/j.diagmicrobio.2007.07.007

12. Thomson RB, Miller JM. Specimen collection, transport, and processing: bacteriology. In: Murray PR, Baron EJ, Jorgensen JH, Pfaller MA, Yolken RH, editors. Manual of Clinical Microbiology. Washington DC: ASM Press; 2003:286-330.

13. Clinical and Laboratory Standards Institute. Performance Standards for Antimicrobial Susceptibility Testing. 30th ed. Wayne, PA, USA: Clinical and Laboratory Standards Institute; 2020.

14. EUCAST. Breakpoints tables for interpretation of MICs and zone diameters 2019. Available from: www.eucast.org. accessed December $18,2019$.

15. Elhadi N, Aljindan R, Aljeldah M. Prevalence of nontyphoidal Salmonella serogroups and their antimicrobial resistance patterns in a university teaching hospital in Eastern Province of Saudi Arabia. Infect Drug Resist. 2013;6:199-205. doi:10.2147/IDR.S51184
16. Ince OT, Yalcin SS, Yardakok K, et al. Salmonella gastroenteritis in children (clinical characteristics and antibiotic susceptibility): comparison of the years 1995-2001 and 2002-2008. Turk J Pediatric. 2012;54(5):465-473.

17. Chang Y-J, Chen Y-C, Chen N-W, et al. Changing antimicrobial resistance and epidemiology of non-typhoidal Salmonella infection in Taiwanese children. Front Microbiol. 2021;12:648008. doi:10.3389/fmicb.2021.648008

18. Lo H-Y, Lai F-P, Yang Y-J. Changes in epidemiology and antimicrobial susceptibility of nontyphoid Salmonella in children in southern Taiwan, 1997-2006. J Microbiol Immunol Infect. 2020;53 (4):585-591. doi:10.1016/j.jmii.2018.06.004

19. Gordon MM, Graham SM, Walsh AL, et al. Epidemics of invasive Salmonella enterica serovar enteritidis and $S$. enterica serovar typhimurium infection associated with multidrug resistance among adults and children in Malawi. Clin Infect Dis. 2008;46(7):963-969. doi:10.1086/529146

20. Meakins S, Fisher IST, Berghold C, et al. Antimicrobial drug resistance in human nontyphoidal Salmonella isolates in Europe 20002004: a report from Enter-net International Surveillance network. Microb Drug Resist. 2008;14(1):31-35. doi:10.1089/mdr.2008.0777

21. Poupart M-C, Chanal C, Sirot D, Labia R, Sirot J. Identification of CTX-2, a novel cefotaximase from a Salmonella mbandaka isolate. Antimicrob Agents Chemother. 1991;35(7):1498-1500. doi:10.1128/ AAC.35.7.1498

22. Rotimi VO, Jamal W, Pal T, Sovenned A, Albert MJ. Emergence of CTX-M-15 type extended-spectrum beta lactamase-producing Salmonella spp. in Kuwait and the United Arab Emirates. J Med Microbiol. 2008;57(Pt 7):881-886. doi:10.1099/jmm.0.47509-0

23. Su L-H, Teng W-S, Chen C-L, et al. Increasing ceftriaxone resistance in Salmonellae, Taiwan. Emerg Infect Dis. 2011;17(6):1086-1090. doi:10.3201/eid/1706.101949

24. Cloeckaert A, Chaslus-Dancla E. Mechanisms of quinolone resistance in Salmonella. Vet Res. 2001;32(3-4):291-300. doi:10.1051/ vetres:2001105

25. Su LH, Wu TL, Chiu CH. Development of carbapenem resistance during therapy for non-typhoid Salmonella infection. Clin Microbiol Infect. 2012;18(4):E91-94. doi:10.1111/j.1469-0691.2012.03767.x

26. Liu C-Y, Huang Y-T, Liao C-H, Hsueh P-R. In vitro activities of tigecycline against clinical isolates of Aeromonas, Vibrio, Salmonella species in Taiwan. Antimicrob Agents Chemother. 2008;52 (7):2677-2679. doi:10.1128/AAC.00002-08

27. Jamal WY, John Albert M, Rotimi VO. High prevalence of New Delhi metalo- $\beta$-lactamase-1 (NDM-1) producers among carbapenemresistant Enterobacteriaceae in Kuwait. PLoS One. 2016;11(3): e0152638. doi:10.1371/journal.pone.0152638

28. Threlfall EJ, Ward LR, Skinner JA, Rowe B. Increase in multiple antibiotic resistance in nontyphoidal salmonellas from humans in England and Wales: a comparison of data for 1994 and 1996. Microb Drug Resist. 1997;3(3):263-266. doi:10.1089/ mdr.1997.3.263

29. Hosek G, Leschinsky DD, Irons S, et al. Multidrug-resistant Salmonella serotype Typhimurium-United States, 1996. Morb Mortal Wkly Rep. 1997;46(14):308-310. 


\section{Publish your work in this journal}

Infection and Drug Resistance is an international, peer-reviewed openaccess journal that focuses on the optimal treatment of infection (bacterial, fungal and viral) and the development and institution of preventive strategies to minimize the development and spread of resistance. The journal is specifically concerned with the epidemiology of antibiotic resistance and the mechanisms of resistance development and diffusion in both hospitals and the community. The manuscript management system is completely online and includes a very quick and fair peerreview system, which is all easy to use. Visit http://www.dovepress.com/ testimonials.php to read real quotes from published authors. 